\title{
La sociología de las organizaciones: perspectivas alternativas
}

\author{
Eguzki Urteaga \\ Universidad del País Vasco \\ eguzki.urteaga@ehu.es
}

Resumen: El objetivo de este artículo no es tanto dar cuenta de las teorías clásicas y contemporáneas de la sociología de las organizaciones en Francia como de integrar nuevos enfoques. Que sean recientes o más antiguas, estas teorías tienen en común el becho de renovar la reflexión sobre las organizaciones a través de otras problemáticas $y$ de nuevas referencias. Un primer enfoque percibe la organización como un «montaje heterogéneo» que insiste en la exigencia de justificación de las organizaciones y en la existencia de una pluralidad de regímenes de acción. Una segunda perspectiva concibe la organización como un "campo» que aparece como un sistema estructurado y jerarquizado donde existen dominantes y dominados y que se manifiesta tanto en la Administración pública como en las políticas públicas. Una tercera interpretación asimila la organización a una red, lo que permite a la vez precisar el análisis estratégico y renovar el estudio de las innovaciones. Una cuarta y última interpretación se interesa por los casos límite que constituyen las instituciones totales.

Palabras clave: sociología, organizaciones, perspectivas, renovación.

Abstract: The aim of this article is not so much to account for classical and contemporary theories of the sociology of organizations in France but to integrate new approaches. Whether they are recent or old, these theories have in common the fact that they renew reflection on organizations by focusing on other problems and new references. An initial approach focuses on the organization as a "heterogeneous assembly" that insists on justifying organizations and the existence of numerous systems of action. A second approach conceives organizations as a "field" that appears to be a structured 
Eguzki Urteaga

and hierarchized system peopled by those in control and those who are controlled. They are found in the public administration and in public policies. A third approach likens organizations to networks, which makes it possible to specify the strategic analysis and renew the study of innovations. A fourth and final approach looks into the extreme cases of total institutions.

Keywords: sociology - organizations - perspectives - renewal. 


\section{Introducción}

¿Dónde empieza y dónde se acaba la sociología de las organizaciones? ¿Se trata simplemente de analizar estas agrupaciones organizadas que constituyen las empresas, las administraciones o las asociaciones, cuyo número y tamaño no han cesado de crecer a lo largo del siglo XX y del inicio del siglo XXI, o es cuestión, a través de ello, de desarrollar una reflexión sobre los mecanismos de cooperación y de acción colectiva y, de ese modo, contribuir a pensar la organización social en un sentido más amplio? De hecho, no existe ninguna vida social sin la existencia de organizaciones y de instituciones. En este sentido, comprender las reglas y la lógica del funcionamiento de esta vida colectiva y de las formas de cooperación a las que da lugar constituye el objeto de la sociología de las organizaciones.

El objetivo de este artículo no es dar cuenta de las teorías clásicas y contemporáneas de la sociología de las organizaciones en Francia, sino integrar nuevos enfoques. Efectivamente, este trabajo parte de la constatación de que la sociología de las organizaciones actual tiene dificultades para renovar sus perspectivas, por lo que puede ser interesante buscar en otros ámbitos, en respuesta a otras preocupaciones, los elementos susceptibles de contribuir a su renovación — por ejemplo, pensar las organizaciones gracias a otras nociones que difieren de las de poder o de cultura-. Asimismo, el concepto de sistema de acción concreto (SAC), forjado por Michel Crozier y Erhard Friedberg (1977), no agota completamente el análisis de las relaciones entre las organizaciones y su entorno. En este sentido, la sociología de las organizaciones se enriquecería si fuese capaz de explorar otras nociones.

Que sean recientes o más antiguas, estas teorías tienen en común el hecho de renovar la reflexión sobre las organizaciones a través de otras problemáticas y de nuevas referencias. Un primer enfoque percibe la organización como un «montaje heterogéneo» que insiste en la exigencia de justificación de las organizaciones y en la existencia de una pluralidad de regímenes de acción. Una segunda perspectiva concibe la organización como un «campo» que aparece como un sistema estructurado y jerarquizado donde existen dominantes y dominados y que se manifiesta tanto en la Administración pública como en las políticas públicas. Una tercera interpretación asimila la organización a una red, lo que permite a la vez precisar el análisis estratégico y renovar el estudio de las innovaciones. Una cuarta y última interpretación se interesa por los casos límite que constituyen las instituciones totales. 


\section{La organización como «montaje heterogéneo»}

La perspectiva iniciada por Luc Boltanski y Laurent Thévenot, así como los trabajos realizados en su estela, resultan especialmente estimulantes para aclarar las lógicas de acción que caracterizan los universos organizativos. Si la noción no es extranjera a la sociología clásica de las organizaciones, bien está fuertemente determinada por las nociones de interés y de estrategia, bien conduce a la segmentación de las organizaciones en dimensiones económicas, sociales, culturales o técnicas. En el primer caso, las lógicas de acción se reducen a estrategias de actor, a la diversidad de los intereses que los animan y que se enfrentan en el seno de un sistema de acción, mientras que, en el segundo caso, las diferentes dimensiones de la acción están yuxtapuestas sin que se comprenda verdaderamente de qué manera los actores se acomodan de esta separación. La vía abierta por Boltanski y Thévenot conduce a precisar las diferentes modalidades de la acción en función de las situaciones atravesadas por los actores que problematizan sus relaciones.

Boltanski y Thévenot (1991) se han interesado en primer lugar por las lógicas de acción que gozan de fuerte legitimidad, públicamente justificables, y que corresponden, en ese sentido, a un régimen de justificación, antes de interesarse por los regímenes de acción más diversificados. Enfrentándolo a varios objetos empíricos, este marco de análisis permite aprehender las organizaciones como lugares de paso, de tensión y de articulación entre lógicas y regímenes de acción de naturaleza diferente. La noción de sistema, que se encontraba hasta entonces en el corazón de la sociología de las organizaciones, deja lugar a la de «montaje heterogéneo».

\subsection{La exigencia de justificación de la organización}

$¡$ ¿El interés y las estrategias de poder constituyen el primer motor de la acción en el seno de las organizaciones, como lo sugiere la sociología inspirada en Crozier? El enfoque propuesto por Boltanski y Thévenot pone el énfasis en situaciones, en el seno de los universos organizativos y más generalmente del mundo social, en las que los actores se ven obligados a demostrar el fundamento de lo que dicen $y$ hacen, de las decisiones que toman y de las acciones que llevan a cabo. Avanzan la hipótesis de que los actores se ven obligados a apoyarse en un número limitado de referentes generales que constituyen principios de justificación y de valoración. Estos pueden ser movilizados tanto para justificar una acción emprendida como para criticar o denunciar una injusticia. La legitimidad de estos referentes resulta de su generalidad y de su universalidad, así como de su ajuste a los dispositivos concretos de las situaciones en las cuales son movilizados si no quieren ser desca- 
lificados. Efectivamente, lejos de ser principios abstractos, suponen una puesta a prueba de la valoración y de la justificación avanzada.

Boltanski y Thévenot han distinguido seis principios de justificación. Estos principios no corresponden a esferas separadas de la sociedad, sino que coexisten en el seno de un mismo espacio o en una misma organización. Su número no está necesariamente fijado una vez y para siempre (Lafaye y Thévenot, 1993), sino que está vinculado al enfoque elegido por los autores, que prefieren principios que responden a exigencias comunes. Efectivamente, si la identificación empírica de estos principios de justificación es indispensable y constituye un indicador serio, no es suficiente para dar cuenta de su universalidad.

El primer principio, el de la inspiración, se fundamenta en la creatividad, la imaginación y la espontaneidad. El principio doméstico se basa en la tradición y en la confianza en las relaciones cuyo único juez es el tiempo. Así, la preocupación por tener colaboradores leales y de confianza justifica su contratación basada en recomendaciones personales. El principio de renombre o de fama está basado en el reconocimiento del mayor número y el crédito concedido por la opinión pública. Por ejemplo, la búsqueda de notoriedad está en el centro de las acciones de comunicación externa e interna emprendidas por numerosas organizaciones. El principio cívico está encarnado por la voluntad general e implica el inicio de acciones colectivas orientadas hacia el interés general y la solidaridad. La empresa se apoya sobre este tipo de legitimidad para justificar las acciones llevadas a cabo a favor de los más desprovistos o del medio ambiente. El principio mercantil se fundamenta en la competencia y en la armonía entre la oferta y la demanda, tales como las que prevalecen en el mercado. Por último, el principio industrial está basado en la eficacia, la productividad y la previsibilidad.

Por lo tanto, las organizaciones no obedecen a un principio único de legitimidad, incluso si, hoy en día, la justificación mercantil tiende a sustituirse a la justificación industrial y a los modelos de planificación y de mejora de la productividad que lo acompañaban. Thévenot (1993) observa que esta activación o reactivación de los vínculos mercantiles no es suficiente para dar cuenta de la dinámica de las organizaciones, las cuales corresponden más a un «montaje heterogéneo» entre exigencias plurales. Por ejemplo, la necesidad, para una empresa, de ser sensible a la demanda de sus clientes se enfrenta a la planificación de la producción o las relaciones de confianza y de fidelidad establecidas con los proveedores se enfrentan del seguimiento de la versatilidad del mercado (EymardDuvernay, 1987). Las tensiones que resultan de estas exigencias múltiples hacen referencia a exigencias más fundamentales y no a simples enfrentamientos entre grupos o servicios cuyos intereses son diferentes, como sería el caso entre los 
servicios comerciales y los servicios de producción o los servicios encargados de la compra. En la continuidad de los estudios llevados a cabo por Alvin Gouldner (1954) se ha demostrado de qué manera una reorganización de los servicios municipales aspiraba a imponer un imperativo de productividad y de racionalidad técnica (justificación de naturaleza industrial) al conjunto del funcionamiento municipal descalificando sistemáticamente la contratación por cooptación (justificación doméstica) o las acciones de solidaridad hacia empleados en dificultad (principio de solidaridad cívica).

Pero, para que una organización se mantenga, el enfrentamiento no puede ser la única forma de relación entre las diferentes lógicas. El compromiso constituye otra forma. Las empresas asociativas estudiadas por Emmanuelle Marchal (1992) combinan en su seno la profesionalidad de la empresa y las exigencias democráticas características del estatus asociativo. El autor muestra la diversidad de las formas organizacionales que resultan de todo ello y de qué manera, según los casos, las exigencias propias al dispositivo asociativo pueden entorpecer o favorecer los imperativos del dispositivo empresarial. Observaciones similares han sido realizadas sobre los agentes bancarios (Wissler, 1989) y los centros educativos (Dérouet, 1992). Los compromisos entre lógicas de acción diferentes pueden a veces tomar la forma de innovaciones técnicas, como cuando se inventa, en una empresa lechera, un autómata susceptible de fabricar camembert tradicional reproduciendo el gesto secular del moldeador (Boissart y Letablier, 1989). Semejante invento permite llegar a un acuerdo entre las exigencias fundamentadas en la tradición y los imperativos de estandarización industrial.

El marco de análisis de la justificación, forjado por Boltanski y Thévenot, abre nuevas perspectivas a la sociología de las organizaciones. El carácter plural y a menudo contradictorio de las exigencias de justificación hace que las organizaciones ya no procedan de una dinámica de ajuste único, como cuando esta se fundamentaba en la capacidad de los actores de adquirir poder y de utilizar las zonas de incertidumbre o en una coordinación cultural, a la imagen de la sociología de la empresa. No en vano, incluso plurales, las formas de justificación son insuficientes para dar cuenta del conjunto del funcionamiento de las organizaciones. El análisis debe poder abrirse a la identificación de otros regímenes de acción.

\subsection{La pluralidad de los regímenes de acción}

La exigencia de justificación no pesa en cada momento sobre todas las situaciones de la vida social. Esta sigue circunscribiéndose a los momentos en los cuales es necesario responder a la crítica, e incluso anticiparla. Los universos organizativos 
no escapan a esta regla y numerosas acciones son llevadas a cabo refiriéndose a otras lógicas.

Luc Boltanski (1990) distingue otros tres regímenes de acción que se añaden al régimen de la justificación:

1. El régimen de la precisión, que se caracteriza por la rutina. Las personas y las cosas están vinculadas unas a otras en dispositivos estabilizados sin que se active la disputa. Este régimen da cuenta de numerosos momentos del funcionamiento ordinario de las organizaciones: cada uno se dedica a sus actividades, los procedimientos dictan sus conductas y no se cuestionan las decisiones.

2. El régimen de la violencia, marcado por la ruptura de los convenios preestablecidos y por el enfrentamiento entre las distintas fuerzas. Los momentos de crisis en el seno de las organizaciones, como, por ejemplo, las huelgas «salvajes», con ruptura de las negociaciones, secuestro de los miembros de la dirección y desbordamiento de los delegados por la base, se refieren claramente a este régimen.

3. El régimen del agape o del amor, que puede definirse como una atención gratuita prestada a otra persona. Este régimen puede ser útil para dar cuenta de las interacciones fugitivas en el seno de las organizaciones y permite dar coherencia a actos a menudo percibidos como extraños o irracionales. Es el caso cuando un directivo solo percibe en su subordinado que acaba de entrar en su despacho a un ser humano $y$, sin decir una palabra, le pide que se retire a pesar de haberlo convocado para avisarle personalmente de ciertas negligencias profesionales que ha cometido.

Estos últimos dos regímenes constituyen experiencias límite en la vida de las organizaciones, cuya finalidad es controlar los excesos de las personas. Philippe Corcuff y Nathalie Duprez se interesan precisamente por situaciones de este tipo y por la manera según la cual son gestionadas en los hospitales por el personal encargado del cuidado de los pacientes y en las ANPE por los agentes en contacto con los usuarios. Estos dos investigadores analizan la manera según la cual, en las relaciones cara a cara, bien con los enfermos, bien con los desempleados, las enfermeras y los agentes de la ANPE se ven afectados por la desgracia del prójimo. Analizan atentamente las tensiones que aparecen entre la necesidad de tratar equitativamente a cada uno, la compasión suscitada por ciertos usuarios o pacientes y la violencia que amenaza constantemente la tranquilidad aparente. Esta búsqueda permite reinterpretar tanto los análisis psicológicos como el estrés de ciertas profesiones, así como las observaciones de Peter Blau a propósito de «las reacciones emocionales», «los sentimientos de culpabilidad»o «una implicación 
excesiva en la ayuda de los usuarios». En este sentido, la investigación empírica resulta fundamental para precisar las modalidades de paso de un régimen de acción hacia otro.

Otros regímenes de acción han sido identificados. El régimen de la familiaridad, identificado por Laurent Thévenot (1994), permite dar cuenta de estas relaciones de proximidad que no tienen un alcance general de justificación. Es el caso de dos compañeros con actividades extraprofesionales comunes que se ponen de acuerdo para compartir el mismo despacho o para tener los mismos horarios. El carácter heurístico de este régimen resulta del hecho de que engloba tanto los vínculos de amistad que crean los actores como la relación privilegiada que el obrero mantiene con su máquina. Este régimen permite no disociar elementos que pertenecen a la sociología de las organizaciones de aquellos tratados preferentemente por la sociología del trabajo.

Justificación, rutina, violencia, compasión, familiaridad, etc., dejan de lado una dimensión fundamental de la acción sobre la cual la sociología inspirada en Crozier había puesto el énfasis, es decir, el carácter estratégico de la acción. El régimen de acción táctico-estratégica colma esta laguna tomando en consideración comportamientos estratégicos y confiriéndoles una validez más restringida que el análisis estratégico. Efectivamente, solo constituye una modalidad de compromiso posible de la acción. Este régimen se manifiesta a través del mantenimiento de una visión estratégica a largo plazo y una capacidad táctica para aprovechar la oportunidad cuando se presenta. Permite, por ejemplo, renovar el análisis de los procesos de decisión en el seno de las organizaciones proponiendo una interpretación renovada de las relaciones entre decisión e implementación (Lafaye, 1994). Además, este régimen está articulado en torno a la justificación en la medida en que el horizonte al que se refiere es públicamente justificable, mientras que las capacidades tácticas desplegadas en esta óptica no lo son necesariamente.

Tanto la identificación de diferentes principios de justificación como la exploración de varios regímenes de acción contribuyen, por lo tanto, a relativizar los modelos estratégicos de la acción. Pero la vía abierta va más allá de la simple constatación de las lógicas de acción: invita a pensar sus relaciones a través de la toma en consideración de los pasos y de los cambios de un régimen a otro, que constituyen la vida ordinaria del funcionamiento de las organizaciones. De este modo, semejante enfoque ya no es compatible con la noción de sistema, casi indisociable de la sociología de las organizaciones. La de «montaje heterogéneo» resulta más pertinente para dar cuenta de la fluidez de los universos organizativos y de los estados múltiples en los cuales estos últimos están comprometidos, así como de la estabilización de los recursos y de la repetición de los comportamien- 
tos. La noción de sistema presupone, incluso en sus concepciones más atenuadas, una coherencia y un estado de equilibrio ausentes en el «montaje heterogéneo». Si esta última noción se sobrepone fácilmente a la de organización, las modalidades de la acción a las que hace referencia conciernen igualmente a las relaciones establecidas con el entorno. Según las situaciones, estas relaciones hacen un llamamiento a las acciones de naturaleza táctico-estratégica, inscribiéndose en dispositivos estabilizados, están sometidas a imperativos de justificación, se rompen en la violencia, etc. Pero hay otras maneras de renovar el análisis de los vínculos entre las organizaciones y su entorno.

\section{De la organización al campo}

Pierre Bourdieu ha forjado la noción de «campo» para definir universos sociales extremadamente variados: mundo del arte o de la literatura, universo científico, ámbito político o administrativo. No en vano, estos universos no asisten únicamente al enfrentamiento de los agentes sociales, sino también al de las organizaciones que persiguen objetivos diferentes. En este sentido, ¿la noción de campo puede contribuir a aclarar las relaciones que mantienen las organizaciones con su entorno próximo, incluso a una mejor comprensión de su funcionamiento interno? Se trata, en un primer momento, de presentar las principales características de los campos sociales mostrando en qué estos últimos se diferencian del concepto de sistema. En un segundo momento, es cuestión de ilustrar el poder heurístico de la noción de campo para el análisis de las organizaciones a través del ejemplo del campo administrativo.

\subsection{El campo social}

La noción de campo tiene las siguientes características (Bourdieu, 1980):

- Un campo es un espacio estructurado y, por lo tanto, jerarquizado de posiciones o de plazas cuyas características son relativamente independientes de sus ocupantes.

- Cada campo se define por retos e intereses específicos irreducibles a los de un campo: lo que hace correr a un científico no es lo que hace correr a un empresario o a un religioso.

- Un campo implica igualmente la posesión o la constitución de un capital propio. Tener un fuerte capital económico es fundamental en el ámbito del negocio, pero totalmente incongruo en el campo científico, donde el 
capital pertinente es de otra naturaleza: una tesis, publicaciones de alto nivel, reconocimiento internacional, etc.

- Un campo necesita que los agentes sociales dotados de disposiciones apropiadas (que Bourdieu denomina habitus) inviertan en su seno, lo que supone conocer las reglas de juego del campo considerado.

- La estructura de un campo es el resultado, en un momento determinado, de la relación de fuerzas entre agentes e instituciones que ocupan posiciones diferentes.

- Un campo es también un espacio dinámico en el cual se producen conflictos para mantener o modificar el estado de la relación de fuerzas: ocupar las posiciones dominantes, transformar las posiciones dominadas en posiciones dominantes, estabilizar las posiciones inestables, hacer reconocer las posiciones situadas en las fronteras del campo, descalificar otros campos, etc. Estas luchas participan en la evolución de la estructura del campo. Disciplinas como la homeopatía o la acupuntura, que se han situado durante un largo período en los márgenes del campo médico, han conseguido hoy en día su reconocimiento e integración.

Cierto número de rasgos tienden a aproximar el concepto de campo al de sistema de acción concreto: 1) ambos designan espacios que no se sobreponen a la noción de organización; 2) estos espacios no son estáticos, sino dinámicos; y 3) tanto los campos como los sistemas de acción concretos están estructurados por relaciones y retos de poder o, más exactamente, por relaciones de fuerza y de dominación.

A pesar de estas similitudes, una primera diferencia se refiere al hecho de que la concepción de la relación de fuerzas en Bourdieu no cubre la definición de las relaciones de poder propuesta por Michel Crozier y Erhard Friedberg. Para estos últimos, la definición muy genérica del poder como control de la incertidumbre está asociada a la diversidad de configuraciones empíricas en el seno de cada sistema de acción concreto. Para Bourdieu, la relación de fuerzas y la dominación están siempre mediatizadas por el reparto desigual del capital propio a cada campo. La noción de capital, que puede definirse como los recursos específicos de cada campo, constituye un nivel intermedio entre los actores y las luchas en las que participan. Por lo tanto, un campo está parcialmente preestabilizado, mientras que un sistema de acción concreto «es el fruto de lo que los actores hacen o han hecho» (Friedberg, 1993: 225). La noción de campo supone, así pues, un cierto nivel de institucionalización, una inscripción en el tiempo, aunque sea únicamente porque las posiciones que estructuran el campo son relativamente independientes de sus ocupantes. 
Una segunda diferencia hace referencia al hecho de que un campo se caracteriza por un consenso mínimo, soporte y condición de las luchas que se desarrollan en su seno. Así, las rivalidades internas en el campo de las Grandes Escuelas se fundamentan en la creencia compartida en la necesidad de formar una élite intelectual. No sucede lo mismo en los sistemas de acción concretos, sobre los cuales Friedberg dice que no presuponen la existencia de una visión compartida o de un acuerdo fundador.

Si el campo implica un nivel de institucionalización y se fundamenta en un consenso mínimo del que no está dotado el sistema de acción concreto, no presupone, a diferencia de este último, mecanismos de regulación que conducen a un estado de equilibrio. La noción de campo, a diferencia de la de sistema, no tiene connotaciones funcionalistas. Si Pierre Grémion (1976) tiene cierta tendencia a considerar que el sistema político-administrativo local permite realizar, entre el Estado y la sociedad civil, ajustes que las reglas no consiguen asegurar por si solas, la noción de campo territorial forjada por Pierre Bourdieu (1990) conduce a cuestionar la existencia de semejantes «ajustes», que dejan de lado la parte fundamental de los ciudadanos para concentrarse en los notables.

Por último, la noción de campo se distingue de las de organización y de sistema de acción porque problematiza la noción de fronteras como un reto constante objeto de luchas entre los actores concernidos. Incluso los trabajos que, en la senda de Michel Crozier, han abordado la ambigüedad de las fronteras organizacionales no han percibido este aspecto, ya que la noción de sistema de acción concreto los conducía a focalizarse en los fenómenos de interpenetración entre las organizaciones y su entorno exterior. No obstante, esta característica de los campos sociales constituye una pista de investigación especialmente estimulante para comprender el funcionamiento de ciertas organizaciones. Así, los partidos políticos y los sindicatos constituyen organizaciones con fronteras de geometría variable, las cuales son objeto de conflictos y de luchas internas para determinar si solamente los militantes que han pagado sus cotizaciones pueden participar en tal decisión o en tal manifestación o pueden hacerlo también los que tienen cierto retraso en el pago, los simpatizantes o incluso los electores. La imposibilidad de organizar unas primarias para designar al candidato de la derecha en la elección presidencial de 1995, como lo habían contemplado varios políticos del RPR y de la UDF, está vinculada a estos conflictos de fronteras y a los retos que estas fronteras representaban para cada uno de estos partidos políticos. Cuando estas luchas carecen de medios para desarrollarse - especialmente porque los partidarios de las posiciones dominantes tienen la capacidad de anular las reacciones de 
los partidarios de las posiciones dominadas-, organizaciones como los partidos políticos o la Iglesia se transforman en aparatos (Bourdieu, 1990).

Además de poner de manifiesto los límites de la noción de sistema de acción concreto, el concepto de campo conduce a evidenciar aspectos de la estructuración del mundo social que pueden ser útilmente transferidos al análisis de los universos organizativos. Permite especialmente dar cuenta de las relaciones complejas - hechas de alianzas, de oposiciones, de competencia- que se tejen de manera duradera entre una serie de organizaciones, las cuales están en el inicio de un gran número de acciones y de decisiones que interesan la sociología de las organizaciones. El ejemplo de la política de la vivienda permite prolongar la reflexión y subrayar el carácter heurístico de la noción de campo.

\subsection{El campo administrativo y la política de vivienda}

¿Cómo se toman las decisiones en materia de financiación de la vivienda o, más ampliamente, cómo se produce la política de vivienda? Cuestiones similares han sido planteadas por la sociología de las organizaciones en general y por el análisis estratégico en particular (Crozier y Friedberg, 1977). Basándose en una investigación llevada a cabo por Pierre Bourdieu y Rosine Christin (1990), se trata de mostrar las aportaciones específicas a un análisis en términos de campo.

Para responder a la cuestión de las modalidades de producción de la política de vivienda y de su financiación, Bourdieu y Christin centran su análisis en un momento delicado. En los años setenta los antagonismos que aparecen entonces conducen a la puesta en marcha de un nuevo orden reglamentario. Este se traduce por el abandono parcial de las ayudas a la construcción concedidas con vistas a favorecer la vivienda social (la ayuda a la piedra dominante desde 1947), que son sustituidas por una ayuda (la ayuda personalizada a la vivienda) concedida a los beneficiarios de viviendas sociales que tienen recursos limitados. Bourdieu y Christin muestran que esta reforma, generalmente imputada a la actividad parlamentaria, resulta de las relaciones de fuerza existentes en un espacio mucho más amplio y complejo. Para dar cuenta de ello, son conducidos a construir el campo de las relaciones de fuerza y de lucha entre, por una parte, agentes y organizaciones administrativos - también ellos en competencia unos con otros- $y$, por otra, instituciones y agentes que, además de ser exteriores al universo administrativo, intervienen para hacer valer sus intereses o los de las personas a las que representan. Es en el seno de este campo que se definen, sobre la base de los antagonismos o proximidades de intereses, así como de las antipatías o afinidades de habitus, los procedimientos que reglamentan el sector inmobiliario. La com- 
plejidad de semejante espacio es perceptible e incluye organizaciones múltiples tales como los ministerios (de Fomento, de Vivienda o de Hacienda), los cuerpos del Estado (Inspección de Finanzas, ingenieros de Puentes), los bancos, las empresas inmobiliarias, los promotores... Las influencias y presiones provienen de todos los sectores.

La complejidad no estriba solamente en el número de organizaciones que intervienen en este espacio, sino en el hecho de que este está constituido por subespacios cuyos retos son específicos. El campo administrativo reúne a los diferentes ministerios, así como a sus servicios exteriores. El campo de la alta función pública se presenta como un subcampo del precedente que goza de una fuerte autonomía. El campo de los organismos HLM (siglas de babitation à loyer modéré, «vivienda de alquiler moderado»), que gestionan el parque de las viviendas sociales, se sitúa en los márgenes del campo administrativo, a pesar de que el de las empresas inmobiliarias le sea exterior. Cada uno de estos campos y subcampos está a su vez atravesado por enfrentamientos y luchas específicos destinados a transformar las relaciones de fuerza existentes. Así, el campo de la alta función pública está marcado por un debate permanente a propósito del rol del Estado que se resume en la alternativa entre intervencionismo y ausencia de intervención. Cada subcampo retraduce, según su propia lógica, las oposiciones que estructuran el espacio que los engloba. Prácticamente cada organización funciona como un campo que está atravesado por luchas de competencia. Comprender de qué manera ha tomado forma una nueva política necesita comprender cómo se organiza este espacio: ¿cuáles son las lógicas que estructuran las acciones y los posicionamientos tanto individuales como colectivos?

La técnica del análisis factorial de las correspondencias es especialmente adecuada para el análisis en términos de campo. Efectivamente, esta técnica permite distinguir las fuerzas en presencia según diversos factores estructurales $y$, de ese modo, facilita la actualización de la estructura de las posiciones que permiten explicar las estrategias de los agentes. En primer lugar, aparece una oposición entre los miembros de la muy alta función pública y los representantes de intereses privados en el sentido amplio, puesto que responsables de entidades públicas de nuevas ciudades, directores de oficinas HLM o cargos electos especializados en las cuestiones de vivienda se encuentran asociados a promotores y banqueros. $\mathrm{Si}$ los agentes del primer polo tienen en común el hecho de pertenecer a los grandes cuerpos estatales y de representar a organizaciones administrativas prestigiosas, los agentes situados en el polo opuesto comparten el hecho de ser ajenos a la alta función pública y carecer de títulos académicos de prestigio. Según el análisis de Bourdieu y Christin, esta primera oposición pone de manifiesto uno de los 
principios fundamentales del campo administrativo: su funcionamiento y sus misiones implican un enfrentamiento con representantes de intereses oficialmente reconocidos pero siempre identificados como privados o particulares, $y$, todo ello, incluso cuando se trata de autoridades públicas (cargos electos locales y nacionales, representantes profesionales y asociativos). Estos últimos están condenados, en cierta forma, a oponerse a una posición subordinada con respecto a los primeros, que tienen el monopolio de la definición del interés general. No en vano, ninguno de estos dos polos es homogéneo y las oposiciones que los atraviesan se traducen de nuevo en sus enfrentamientos en torno a la puesta en marcha de un nuevo orden reglamentario.

El segundo principio de división opone los «financieros» a los «técnicos». Los primeros agrupan a funcionarios provenientes de la Escuela Nacional de Administración (ENA) y de la Inspección de Finanzas en funciones en los gabinetes ministeriales, en el seno del Ministerio de Finanzas o en bancos. Los segundos provienen sobre todo de la Escuela Politécnica o del Cuerpo de Puentes y Calzadas y están vinculados al Ministerio de Fomento. Están asociados a ellos los cargos electos locales y los representantes del movimiento HLM.

El tercer y último principio de división permite identificar un grupo de «innovadores» que no se identifica con ninguna institución u organización y que supera todas las oposiciones precedentes. Sus miembros tienen en común el hecho de tener unas propiedades que les distinguen de su institución de pertenencia: juventud, vínculos con centros de investigación para altos cargos, notoriedad nacional para los cargos electos locales, etc. En el seno del campo administrativo, los innovadores tienen un capital, basado en la técnica y la ciencia, movilizable rápidamente y que se opone al capital burocrático tradicional, hecho de experiencia y de conocimiento de los reglamentos. De este grupo surgirán las fuerzas capaces de superar los antagonismos que paralizan el campo administrativo.

Tras identificar la estructura del campo, Bourdieu y Christin la comparan con los posicionamientos. Estos, ya provengan de organizaciones o de individuos, tienden a repartirse sobre un continuum que va del mantenimiento de la «ayuda a la piedra» a su abandono completo en favor de la ayuda a la persona. Bourdieu y Christin observan que el espacio de los posicionamientos cubre casi perfectamente el espacio de las posiciones. Así, el Ministerio de Finanzas y el Ministerio de Fomento presentan tesis antagónicas. El primero aboga por la sustitución pura y simple de la ayuda a la piedra por la ayuda personalizada a la vivienda, mientras que el segundo, aliado a los organismos HLM y a las constructoras sociales, busca mantener el sistema en vigor. 
El grupo de los innovadores ha permitido superar el statu quo de las oposiciones tradicionales, consiguiendo imponer una solución de compromiso que combina la ayuda a la construcción con la ayuda personalizada. El éxito de este proyecto, que ha consistido en movilizar y conciliar intereses divergentes, se debe parcialmente al hecho de que ocupaban posiciones extremadamente dispersas en el seno del campo.

$\mathrm{El}$ análisis en términos de campo, propuesto por Bourdieu, permite reconstituir el conjunto del espacio pertinente en el seno del cual se ha producido la transformación de la política de la vivienda en la mitad de los años setenta. Este aspecto no se reduce al campo administrativo, sino que incluye organizaciones que le son exteriores. La noción de campo permite una identificación más amplia de la lista de los actores pertinentes y del conjunto de las relaciones de alianza y de oposición que sirven para determinar las interacciones y los posicionamientos. Mientras que el análisis estratégico se limita a señalar que los actores pertenecen a varios sistemas y que pueden desplazar lo que está en juego de un sistema a otro (Friedberg, 1993:234), el análisis en términos de campo permite pensar los encajamientos de los diferentes espacios y constituye un poderoso instrumento para pensar las relaciones complejas que se establecen entre diferentes organizaciones y la manera según la cual estas relaciones están parcialmente determinadas por los espacios más amplios que las engloban.

La noción de red dibuja otra arquitectura de las relaciones internas y externas de las organizaciones.

\section{De la organización a la red}

La sociología de las organizaciones empieza en Francia a interesarse por el análisis de las redes (Lezaga, 1994). Este último es presentado por Alain Degenne y Michel Forsé (1994) como un nuevo paradigma de las ciencias sociales. La perspectiva, claramente inductiva, intenta dar cuenta de las relaciones que unen entre sí actores y unidades (hogares, familias, empresas, naciones..+). Este paradigma, que conoce un éxito creciente en varias disciplinas, cubre diversos ámbitos de las ciencias sociales y humanas, desde las redes de parentesco hasta las relaciones internacionales. Se acompañan frecuentemente de modelizaciones matemáticas. Su interés es múltiple para la sociología de las organizaciones. 


\subsection{Una profundización del análisis estratégico}

El análisis de las redes, según Emmanuel Lazega, permite estudiar tanto el funcionamiento interno de las organizaciones como las relaciones que mantienen con su entorno. A nivel interno, el análisis de las redes permite describir los vínculos de amistad, de asesoramiento o de influencia que atraviesan las fronteras formales trazadas por la organización. Así, en el seno de una gran empresa industrial, esta perspectiva ha permitido, a través del tratamiento de archivos sobre un período de treinta años, identificar la estructura de las relaciones entre los miembros que componen la organización, sus ramificaciones y su evolución. El análisis hace aparecer una polarización entre dos grandes bloques antagónicos, los cuales controlan las contrataciones realizadas en función de las recomendaciones. Una tercera posición, ocupada por miembros que no están implicados en ninguno de los dos bloques precedentes, asegura la integración frágil de la empresa, la cual no resistirá a conflictos internos que se traducen en el despido de los líderes del polo intermedio. Este ejemplo muestra que el análisis de las redes puede ser un instrumento susceptible de ayudar a la identificación precisa y cuantificada de los mecanismos de regulación subyacentes.

El análisis de las redes se interesa igualmente por los vínculos existentes entre las organizaciones y por la naturaleza de los recursos que circulan entre ellas, mostrando que estas relaciones se caracterizan por un importante número de intercambios no económicos. Pueden identificarse tres ejes principales. El primero analiza la formación y el mantenimiento de las redes que pasan por la presencia de los mismos individuos en el consejo de administración de varias empresas. La segunda dirección estudia la influencia de las redes de lobbying (grupos de presión) sobre la definición de las políticas públicas. El tercer eje relaciona las posibilidades de supervivencia de las empresas competidoras y los vínculos informales que establecen entre sí para gestionar las incertidumbres vinculadas a la demanda.

Según Lezaga, las afinidades entre el análisis de las redes y el análisis estratégico estriban, en primer lugar, en el hecho de que ambos obedecen a un enfoque inductivo: en ambas perspectivas tanto el sistema como los actores colectivos presentes o la configuración de sus relaciones no constituyen una realidad observable, sino que resultan de la investigación empírica. En segundo lugar, el análisis de las redes permite describir en los mismos términos las redes formales e informales, intraorganizacionales o bien interorganizacionales, y permite identificar de qué manera ambas dimensiones orientan la acción y definen un sistema de acción en el seno del cual los actores desarrollan sus estrategias. El análisis puede poner de manifiesto la manera según la cual redes de relaciones informales sustituyen 
las carencias de la estructura formal, pero también de qué manera esta determina los comportamientos informales.

Además, Lezaga muestra que el análisis de redes se ajusta especialmente a la definición propuesta por Crozier del poder como control de las zonas de incertidumbre. Efectivamente, permite identificar a los actores centrales de un sistema de análisis concreto recurriendo a medidas de centralidad o de prestigio. Así, cuando un actor establece un número importante de vínculos con otros actores, puede establecer esos lazos rápidamente, se convierte en un paso obligado para otros y adquiere cierta centralidad. Un alto nivel de centralidad puede considerarse como un indicador de extensión o del carácter crucial de la incertidumbre controlada por el actor.

Por último, el análisis de las redes y el análisis estratégico tienen en común el hecho de interesarse por la dinámica de las estructuras organizativas. En primer lugar, el análisis de las redes resulta útil para identificar las relaciones entre sistemas de acción de niveles diferentes, punto débil del análisis estratégico en comparación con los análisis en términos de campo. Efectivamente, permite pasar del nivel intraorganizacional reconstituyendo, por aproximaciones sucesivas, el sistema de acción de nivel superior y contribuyendo a identificar las articulaciones entre lo individual, lo grupal, lo organizacional y lo interorganizacional. Además, el análisis de redes puede resultar valioso para analizar procesos dinámicos e innovaciones en el seno de las organizaciones. Desde esta perspectiva, aunque la sociología de la innovación, iniciada por Michel Callon y Bruno Latour (1995), hace igualmente un llamamiento a la noción de red, no se confunde por ello con el análisis estratégico (Bernoux, 1995: 160).

\subsection{Una renovación del análisis de la innovación}

Se admite generalmente que el carácter dinámico de las organizaciones y su desarrollo dependen en gran medida de sus capacidades para innovar. Michel Callon y Bruno Latour, poniendo de manifiesto las condiciones de producción y de circulación de las innovaciones técnicas y de los conocimientos científicos, aportan una respuesta original a esta cuestión. Demuestran que la elaboración de las innovaciones ignora las fronteras organizacionales, las de los laboratorios (Latour y Woolgar, 1988). La reimplantación de las conchas Saint-Jacques en la bahía de Saint-Brieuc, estudiada por Michel Callon (Callon, 1986; Callon y Law, 1989), servirá de hilo conductor a nuestra exposición.

A principios de los años setenta las conchas Saint-Jacques habían desaparecido de la bahía de Brest como consecuencia de los predadores y de la pesca excesi- 
va. Una amenaza similar pesaba sobre la bahía de Saint-Brieuc. Tres investigadores del CNEXO (Centro Nacional de Explotación de los Océanos) propusieron entonces un programa de investigación destinado a identificar las condiciones en las que una técnica japonesa de cría de conchas Saint-Jacques podría adecuarse al territorio francés (lo que no era evidente, ya que las conchas de Saint-Brieuc son de otra especie que las japonesas). Michel Callon muestra que el lanzamiento de este programa y su éxito pasaban por una asociación inédita entre múltiples actores: la comunidad científica, los pescadores, los poderes públicos y las conchas de Saint-Brieuc. Lejos de ser endógena, la producción de conocimiento necesitaba la movilización y la cooperación de todos estos actores. Para interesar a cada uno de estos actores en este proyecto, los tres investigadores del CNEXO realizaron lo que Callon denomina una serie de traducciones. Así, lo que para el conjunto de la comunidad científica era una cuestión de conocimiento fundamental, debía ser traducido en términos de supervivencia económica para los pescadores, en problema de perpetuación de la especie para las conchas Saint-Jacques y en términos de imagen para los poderes públicos (ayuntamiento y región).

Traducido de esta forma, el programa de investigación se convirtió en una necesidad para cada uno de estos actores. Los investigadores lo convirtieron en un paso obligado: la existencia de las conchas Saint-Jacques, el honor de la comunidad científica, los ingresos de los pescadores y la notoriedad de la región dependían de ello. Los investigadores llegaron a ser los portavoces de este conjunto heterogéneo en la medida en que eran los únicos capaces de comunicar estos universos separados. Pero traducir es igualmente desplazar. Efectivamente, la movilización de los diferentes actores se acompañó de una serie de desplazamientos e incluso de redefiniciones identitarias. Así, las larvas de las conchas Saint-Jacques, dispersadas y movidas en función de las corrientes marinas, eran recogidas y mantenidas en un dispositivo concebido para ello. Posteriormente sus movimientos eran traducidos en medidas, curvas y gráficos para la comunidad científica. Asimismo, los pescadores se convertían en un primer momento, respondiendo a la solicitud de los investigadores, en observadores atentos de los movimientos de las larvas y realizaban extracciones. Posteriormente se convirtieron en criadores.

De estas asociaciones inéditas y de las operaciones de traducción a las que dieron lugar para que la innovación tomara forma emergió una red sociotécnica. Puede definirse como una serie de procesos entre actores humanos (investigadores, pescadores, financiadores, etc.) y no humanos (conchas Saint-Jacques, larvas, corrientes marinas, instrumentos de medida, etc.) que se encuentran interrelacionados. La noción de red, tal y como ha sido concebida, pone de manifiesto la 
extensión considerable de las relaciones y las asociaciones necesarias para la producción de innovaciones. Por una parte, evidencia el hecho de que el desarrollo de las innovaciones implica la transgresión de las fronteras organizacionales: el mundo exterior ya se encuentra en el corazón del laboratorio o del servicio de desarrollo, cuyo éxito depende de su capacidad de introducir nuevos actores (Chateauraynaud, 1991). Por otra, y contrariamente a las concepciones más usuales del análisis de redes, son inclusivas, ya que personas y entidades están vinculadas entre sí por relaciones variadas: relaciones de subordinación y de intercambio, relaciones técnicas y financieras, pero también relaciones químicas y biológicas. Los actores humanos y no humanos contribuyen así a definirse mutuamente, es decir, a precisar mutuamente su identidad, sus intereses y sus relaciones, que no están fijados una vez para siempre. Por lo tanto, la lista de los actores pertinentes está abierta, lo que la noción de sistema de acción no permite generalmente tomar en consideración.

Pero la noción de red permite igualmente pensar la estabilización de estos recursos y relaciones heterogéneos. Mientras que la noción de asociación ponía el énfasis en un proceso en vía de constitución, la de red designa el resultado más o menos solidificado de cadenas de traducción. Estas constituyen la infraestructura mínima para que se produzca un conocimiento o una innovación. Así, numerosas inversiones que concernían tanto a las conchas Saint-Jacques a como los pescadores fueron necesarias: la sociedad local, la fauna local y la fauna marina fueron puestas en marcha para que al final el laboratorio pudiera instalarse y se produjera conocimiento (Callon, 1989: 24).

Además, las cadenas de traducción, que ponen en equivalencia recursos heterogéneos, no se descomponen necesariamente con la aparición de la innovación, ya que se crean vínculos y circuitos. Las redes aparecen, de ese modo, engarzadas unas con otras. Enrolar un nuevo actor significa a menudo movilizar la red a la que está conectado, como lo muestra el reto que representa la contratación de colaboradores para numerosas organizaciones. Desde esta perspectiva, el laboratorio desempeña un rol de catalizador de las diferentes redes que el descubrimiento o la innovación agrega. Delimita, organiza, gestiona y transforma el entorno, precisa Callon (1989: 211-212), el cual le asigna unos márgenes de maniobra determinados. Contemplados así, los laboratorios o las unidades en que se producen innovaciones son entidades fluctuantes que se deforman con las redes y los actores-red que los atraviesan y estructuran.

La vía abierta por Michel Callon y Bruno Latour permite repensar la cuestión de la innovación en los universos organizacionales, a menudo analizados a través del modelo simplista de la difusión. La noción de red sociotécnica transforma 
completamente ese concepto. Poniendo el énfasis en la heterogeneidad de los recursos y las relaciones necesarios para la producción de innovaciones, enriquecen el análisis de las redes y relativizan tanto la noción de organización como la de sistema.

\section{Las instituciones totales}

Los enfoques más clásicos de las organizaciones, a la imagen de las que contribuyen a su renovación, tienen en común el hecho de considerar las organizaciones como universos abiertos sobre el mundo que las rodea. Los análisis en términos de campo o de red, más aún que los de sistema, contribuyen a hacer estallar la noción de organización en beneficio de espacios de relaciones que las superan. No obstante, esta tendencia del análisis no debe hacernos olvidar que existen organizaciones que, por el contrario, tienden a funcionar en universos cerrados. A principios de los años sesenta Erving Goffman publica un libro, Asilo, centrado en un tipo específico de organizaciones, las que intentan instaurar fronteras estancas entre sus miembros y el mundo exterior. Goffman había trabajado un año en el seno de un hospital psiquiátrico y las observaciones recogidas constituyen el material empírico de su análisis de las instituciones totales (Goffman, 1968).

\subsection{Las instituciones totales}

Por el concepto de institución total, Goffman entiende «un lugar de residencia y de trabajo donde un gran número de individuos, situados en la misma situación, cortados del mundo exterior por un período relativamente largo, llevan juntos una vida reclusa cuyas modalidades están explícita y minuciosamente regladas» (Goffman, 1968: 41), como en el caso de los hospitales psiquiátricos, de las cárceles o de las comunidades religiosas. La reclusión constituye el rasgo fundamental de las instituciones totales, que instauran, para ello, una separación entre los miembros de la organización (los reclusos) y el entorno social exterior del que forma parte la dirección. Goffman detalla las técnicas que contribuyen a la pérdida de autonomía de los reclusos: mortificación, despersonalización e instauración de un sistema de privilegios.

Las técnicas de mortificación aspiran a que el recluso se deshaga de su personalidad anterior. Aspiran a introducir una ruptura entre el pasado y el futuro del recluso. La primera de ellas está constituida por el aislamiento: el recluso está cortado del mundo, especialmente de su entorno familiar, las 24 horas del día. 
Las visitas están fuertemente reglamentadas y a veces prohibidas. Las ceremonias de admisión recurren igualmente a técnicas de mortificación en la medida en que consisten casi siempre en privaciones e incluso en humillaciones. Difieren de una institución a otra: registro de identidad, asignación de una matrícula, inventario de las pertenencias personales, sesión de desnudamiento, desinfección, reparto de ropa del centro, ritos de iniciación, novatada, etc. A través de estas fórmulas de admisión, la institución total despoja al nuevo entrante de los objetos y signos de sus pertenencias anteriores y le impone los de su nueva pertenencia. Las marcas corporales, las sevicias y los ultrajes contribuyen igualmente al deterioro de la autoestima de los reclusos y marcan una ruptura con el pasado: tonsura en ciertas comunidades religiosas, gestos y palabras de deferencia en el ejército, golpes y sevicias corporales en ciertas cárceles o campos. Por último, la confesión y la denuncia se erigen generalmente en norma.

Las técnicas de despersonalización marcan una etapa adicional, puesto que intentan romper el vínculo que une al individuo con sus actos. El enrolamiento, que consiste en el control constante de todos los aspectos (psicológicos y físicos) de la vida de los miembros a través de la promiscuidad y la imposibilidad de aislarse, la vigilancia continua, la obligación de participar en las actividades colectivas..., es una de ellas. Otra estriba en la sanción de los medios de defensa contra la institución (enfado, insolencia, ironía, burla, etc.) a los que recurren los reclusos. La instauración de un sistema de privilegios, que tiene como efecto someter completamente al individuo a la institución, viene a completar la panoplia de técnicas que introducen una separación con el mundo exterior. Tal sistema está compuesto por una serie de prescripciones, restricciones y prohibiciones a las que el recluso debe someterse, de recompensas o de favores limitados concedidos al recluso como contrapartida a su sumisión mental y física y de sanciones infantilizantes.

¿Cómo se adaptan los reclusos a la institución total? Erving Goffman distingue cuatro grandes modos de adaptación que pueden convivir en un mismo individuo y variar según los momentos y las situaciones: el repliegue sobre sí mismo constituye una forma de descompromiso, la insumisión consiste en desafíos permanentes lanzados al personal de la institución, la instalación se traduce por la acomodación de ciertas obligaciones de la institución y la conversión marca la adopción por los reclusos del punto de vista de la dirección. La película de Milos Forman Alguien voló sobre el nido del cuco escenifica la mayoría de los modos de adaptación: la insumisión está encarnada por el héroe, papel desempeñado por Jack Nicholson, enfrentado a la enfermera jefe, mientras que el indio autista ofrece una clara ilustración del repliegue sobre sí mismo. La vuelta a la vida fuera de la 
institución total, cuando se produce, se traduce en una sensación de resurrección asociada a una fuerte ansiedad debida a los estigmas de la vida en reclusión y a la pérdida de los hábitos diarios exigidos por la vida en sociedad.

$¿$ Qué representaciones adopta el personal de las instituciones totales y cómo trata a los reclusos? Este recurre, según Goffman, a esquemas interpretativos que pretenden racionalizar las características de la institución total. Los reclusos están sistemáticamente identificados a través del objetivo de la institución, ya que un paciente está necesariamente loco, así como un preso es un delincuente. Sin embargo, el personal parece estar sometido a una tensión entre dos maneras antagónicas de tratar a los reclusos. Por una parte, estos son deificados, dado que se trata de expedientes, de matrículas, de seres deshumanizados que circulan de un servicio a otro. Por otra, los reclusos están considerados como personas que deben gozar de cierto trato humano a través de la concesión de atención médica. Esta exigencia de humanidad puede, paradójicamente, conducir a convertir al recluso en un objeto inanimado, como cuando la necesidad de proteger a una persona suicida contra sí misma conduce a encerrarla e incluso a atarla a una cama.

\subsection{Una noción de carácter heurístico}

Una noción solo tiene cierto interés si es retomada y trabajada por otros investigadores y aplicada a otros objetos empíricos. Philippe Bernoux (1981), en su libro Un travail en soi, recurre al concepto de institución total para designar «la institución-empresa» que no concede ninguna autonomía a los trabajadores que la componen. Se trata de una empresa tayloriana que solo reconoce a individuos adscritos a unas máquinas, cambiados autoritariamente de puestos y de grupos de trabajo, sometidos a un ritmo impuesto, desposeídos de cualquier capacidad para comunicarse. Bernoux encuentra en el universo cerrado de los obreros especializados los principales rasgos identificados por Goffman. No en vano, el análisis sigue siendo incompleto ya que pone el énfasis en lo que separa la empresa de la institución total tal y como lo ha construido Goffman.

La investigación llevada a cabo por Jeannine Verdès-Leroux (1981) sobre el Partido Comunista Francés (PCF) aparece en este sentido como la más exhaustiva. La noción de institución total es utilizada para analizar las prácticas del PCF, ordenar el conjunto de los hechos más significativos y mostrar su lógica. Toda una serie de conductas tanto de los dirigentes como de los militantes de base son similares a las que existen en las instituciones totales: disponibilidad absoluta; ritos de iniciación; instauración de barreras con el exterior a través de prohibiciones que se refieren a ciertos lugares, prácticas o gustos; conversión a la 
ideología comunista; modificación de la imagen de sí mismo a través de la autocrítica y la denuncia realizada en nombre de la vigilancia revolucionaria; control generalizado del pensamiento y de la vida privada, incluso amorosa; sistema de privilegios basado en elogios y viajes a la URSS; estigmatización de la salida, que se identifica con la traición, etc. Simultáneamente, Jeannine Verdès-Leroux se interesa por la singularidad del PCF en el seno de las instituciones totales: ausencia de obligaciones y de marcas corporales, adhesión voluntaria de los miembros y orgullo de formar parte del partido. La iniciación es vivida como una etapa feliz y los miembros aceptan todos los aspectos de la vida militante. Por último, el partido está considerado a menudo como una familia solidaria y su fetichización persiste entre los miembros que han roto con el partido.

Jeannine Verdès-Leroux utiliza el concepto de institución total como un tipo ideal y muestra en qué el PCF se aproxima y se aleja de él. Efectivamente, el carácter verdaderamente heurístico del concepto de institución total estriba en su capacidad de informarnos sobre el funcionamiento de cualquier organización. Como complemento de las investigaciones que han puesto de manifiesto las relaciones entre las organizaciones y su entorno, el análisis iniciado por Goffman nos invita a mirar detalladamente los procedimientos a través de los cuales las organizaciones intentan integrar a sus miembros y construir la cohesión interna para, de ese modo, realizar una ruptura, según unos niveles variables, entre el interior y el exterior de la organización. La cultura de empresa, que estaba de moda durante las décadas de 1980 y 1990, se ha traducido en un repliegue sobre sus preocupaciones internas. En este sentido, toma las mismas formas que las instituciones totales.

\section{Conclusión}

En definitiva, los trabajos presentados en este artículo son heterogéneos y dan cuenta de la existencia de teorías y conceptos que permiten renovar el análisis de las organizaciones más allá de los enfoques clásicos. No en vano, la compatibilidad entre las antiguas y las nuevas perspectivas no es evidente, ya que los enfrentamientos activos, las críticas entrecruzadas y las intensas discusiones de las que da cuenta el libro de Friedberg pueden permitir hacer avanzar el conocimiento y, quizás, dar lugar a la constitución de nuevas orientaciones.

Asimismo, la sociología de las organizaciones constituye desde hace varios años un polo especialmente productivo en el seno de las ciencias sociales. La abundancia de las investigaciones y la transgresión frecuente de las barreras disciplinarias pueden a veces dar la sensación de una dispersión del campo y de 
un debilitamiento de su legibilidad. Puesto que el conocimiento no progresa de manera lineal, esta abundancia demuestra una capacidad real de renovarse. Los trabajos que intentan fundar una sociología de la empresa dan cuenta de ello, así como los cuestionamientos provenientes de los marcos conceptuales inicialmente alejados del análisis de las organizaciones. La riqueza de la sociología de las organizaciones estriba igualmente en el hecho de que numerosos autores que han contribuido a su desarrollo aspiran igualmente a la comprensión de fenómenos sociales más amplios. La fuerza de la sociología de las organizaciones proviene precisamente de su capacidad de superar las fronteras de su campo temático.

Se considera a menudo que uno de los recursos de la sociología de las organizaciones estriba en el éxito que tiene en el mundo empresarial. En este sentido, podemos preguntarnos si este éxito no se basa en un malentendido: esta no es, a pesar de ciertos intentos, una ciencia de la gestión. Su primera vocación no es ofrecer soluciones técnicas a los actores ni a los dirigentes de las organizaciones, incluso aunque los conocimientos que produce puedan conducir a reflexionar sobre sus prácticas y las de sus colaboradores y sobre los instrumentos de los que disponen. Su objeto es ante todo la aprehensión y la comprensión de las formas sociales y de los modos de cooperación a los que las personas recurren para llevar a cabo sus acciones. Pero es evidente que la práctica empírica y los protocolos de investigación, especialmente cuando toman la forma de la investigación-acción, traducen cierta permeabilidad entre las ciencias sociales y las preocupaciones de los actores. Esta permeabilidad puede a veces conducir a ciertas ambigüedades entre estos ámbitos, como subraya Marc Maurice (1992: 314-315).

Michel Wieviorka y Sylvaine Trinh (1989: 240) observan igualmente, a propósito de una investigación llevada a cabo con varios directivos de EDF, que los sociólogos están constantemente solicitados como formadores o son percibidos como consultores y que tienen dificultades para mantener su estatus de investigadores. Erhard Friedberg define, por su parte, una práctica de investigación en la cual la producción de conocimiento y su utilización para la acción están íntimamente vinculadas. No se trata de elaborar ideas generales transferibles de un contexto de acción a otro y susceptibles de ser aplicadas concretamente, sino de utilizar conocimientos producidos en un contexto de acción dado para modificar la práctica de los actores y, a la vez, utilizar esta práctica modificada para afinar los conocimientos producidos y abrir nuevos caminos de investigación (Friedberg, 1993). La producción de un saber científico sobre las organizaciones no exige, por lo tanto, una separación clara entre investigación y acción. En todo caso, las investigaciones sociológicas en el seno de las organizaciones suponen controlar rigurosamente los efectos que la presencia y la intervención del investigador pro- 
ducen sobre su campo de investigación y sobre los actores que lo componen. Conducen, por lo tanto, a una práctica de la reflexividad sociológica, es decir, a una toma en consideración, en el análisis, de las relaciones que se producen entre el investigador y el encuestado.

\section{Bibliografía}

Bernoux, P. (1995). La sociologie des entreprises. París: Seuil.

Boissart, P. y Letablier, M-T. (1989). «Un compromis d'innovation entre tradition et stantardisation dans l'industrie laitière», en L. Boltanski y L. Thévenot (coords.) (1989). Justice et justesse dans le travail. Cahiers du CEE-PUF $\$ 209-218$.

Boltanski, L. (1990). L’Amour et la justice comme compétence. París: Metailié.

Boltanski, L. y Thévenot, L. (1991). De la justification. París: Gallimard.

Bourdieu, P. (1980). Questions de sociologie. París: Minuit.

Bourdieu, P. (1990). «Droit et passe-droit. Le champ des pouvoir territoriaux et la mise en œuvre des règlements». Actes de la recherche en sciences sociales, 81-82.

Bourdieu, P+ y Christin, R. (1990)+ «La construction du marché Le champ administratif et la production de la politique du logement». Actes de la recherche en sciences sociales, 81-82:65-85.

Chateauraynaud, F. (1991). «Forces et faiblesses de la nouvelle anthropologie des sciences». Critique, 529-530:459-478.

Callon, M. (1986). «Eléments pour une sociologie de la traduction. La domestication des coquilles Saint-Jacques et des marins-pêcheurs dans la baie de Saint-Brieuc». L'Année sociologique, 36: 169-208.

Callon, M. (1989). La science et ses réseaux. Genèse et circulation des faits scientifiques. París: La Découverte.

Callon, M. y Law, J. (1989). "La proto-histoire d'un laboratoire», en M. CALLON (coord.) (1989). La science et ses réseaux. Genèse et circulation des faits scientifiques, París: La Découverte.

Crozier, M. y Friedberg, E. (1977). Lacteur et le système. París: Seuil.

Derouet, J-L. (1992). Ecole et justice. París: Metailié.

Desmarez, P. (1986). La sociologie industrielle aux États-Unis. París: Armand Colin.

Eymard-Duvernay, F. (1987). «Les entreprises et leurs modèles». Cahiers du CEE-PUF: 5-12.

Forsé, M. (1994). Les réseaux sociaux. París: Armand Colin. 
Friedberg, E. (1993). Le pouvoir et la règle. Dynamiques de l'action organisée. París: Seuil.

Goffman, E. (1968). Asiles. París: Minuit.

Gouldner, A.V. (1954). Patterns of Industrial Bureaucracy.

Grémion, P. (1976). Le pouvoir périphérique. Bureaucrates et notables dans le système politique français. París: Seuil.

LAFAYE, C. (1994). «Aménager un site littoral. Entre politique et pragmatisme». Etudes rurales, 133-134: 163-180.

Lafaye, C. y Thévenot, L. (1993). «Une justification écologique? Conflits dans l'aménagement de la nature». Revue française de sociologie, XXXIV: 495-524.

Latour, B. (1995). La science en action. París: Gallimard.

Latour, B. y Woolgar, S. (1988). La vie en laboratoire. La production des faits scientifiques. París: La Découverte.

Lazega, E. (1994). «Analyse de réseaux en sociologie des organisations», Revue française de sociologie, XXXV: 293-320.

Marchal, E. (1992). «L'entreprise associative entre calcul économique et désintéressement», Revue française de sociologie, XXXIII: 365-390.

Maurice, M. (1992). «Les sociologues et l'entreprise», en R. Sainsaulieu (coord.) (1992). L'entreprise, une affaire de société, París: Presses de Sciences Po.

Mayo, E. (1933). The Human Problems of an Industrial Civilization. Nueva York: Macmillan.

Stroobants, M. (1993). La sociologie du travail. París: Nathan.

Thévenot, L. (1993). «La trame des organisations», en T. Globokar (coord.) (1993). Entreprise, société, communauté, París: Autrement.

Thévenot, L. (1994). «Le régime de la familiarité». Genèses: 17.

Verdes-Leroux, J. (1981). «Une institution totale autoperpétuée. Le parti communiste français». Actes de la recherche en sciences sociales, 36-37:33-63.

Wieviorka, M. y Trinh, S. (1989). Le modèle EDF. París: La Découverte.

Wissler, A. (1989). «Les jugements dans l'octroi du crédit», en L. Boltanski y L. Thévenot (coords.) (1989). Justice et justesse dans le travail, Cahiers du CEE-PUF: 67-120. 